\title{
Existence and uniqueness of nontrivial solutions to a system of fractional differential equations with Riemann-Stieltjes integral conditions
}

\author{
Bingmei Liu' ${ }^{1 *}$, Junling Li ${ }^{1}$ Lishan Liu² and Yongqing Wang ${ }^{2}$
}

\section{"Correspondence:}

lbm2009@cumt.edu.cn

${ }^{1}$ School of Mathematics, China

University of Mining and

Technology, Xuzhou, China

Full list of author information is

available at the end of the article

\section{Springer}

\begin{abstract}
This paper studies a system of fractional differential equations with Riemann-Stieltjes integral conditions. The existence and uniqueness of nontrivial solutions to the above system are established under some weaker conditions by the Leray-Schauder topological degree. Two examples are set up to testify the validity of the main results.
\end{abstract}

MSC: 34B15;47H10;47H11

Keywords: Fractional differential equations; Riemann-Stieltjes integral; Topological degree; Uniqueness

\section{Introduction}

The purpose of this paper is to establish the existence and uniqueness of solutions for the following system of fractional differential equations with Riemann-Stieltjes integral boundary conditions (for short, FBVP):

$$
\left\{\begin{array}{l}
D_{0+}^{\alpha} u(t)+h(t) f(t, v(t))=0, \quad 0<t<1, \\
D_{0+}^{\alpha} v(t)+h(t) g(t, u(t))=0, \quad 0<t<1, \\
u(0)=u^{\prime}(0)=0, \quad u(1)=\int_{0}^{1} u(\tau) d \beta(\tau), \\
v(0)=v^{\prime}(0)=0, \quad v(1)=\int_{0}^{1} v(\tau) d \beta(\tau),
\end{array}\right.
$$

where $2<\alpha \leq 3$ is a real number, $D_{0+}^{\alpha}$ is the standard Riemann-Liouville differentiation, $\beta$ is right continuous on $[0,1)$, left continuous at $t=1$, and nondecreasing on $[0,1]$ with $\beta(0)=0, \int_{0}^{1} u(\tau) d \beta(\tau)$ denotes the Riemann-Stieltjes integral of $u$ with respect to $\beta$. Here the nonlinear terms $f, g:[0,1] \times(-\infty,+\infty) \rightarrow(-\infty,+\infty)$ are continuous sign-changing functions and $f, g$ may be unbounded from below, $h:(0,1) \rightarrow[0,+\infty)$ with $0<\int_{0}^{1} h(s) d s<$ $+\infty$ is continuous and is allowed to be singular at $t=0,1$.

Fractional differential equations play an important role in many engineering and scientific disciplines such as physics, chemistry, aerodynamics, electrodynamics of complex medium, polymer rheology, diffusive transport akin to diffusion, probability, electrical networks, etc. For details, see [1-3] and the references therein. By using a nonlinear alterna-

(c) The Author(s) 2018. This article is distributed under the terms of the Creative Commons Attribution 4.0 International License (http://creativecommons.org/licenses/by/4.0/), which permits unrestricted use, distribution, and reproduction in any medium, provided you give appropriate credit to the original author(s) and the source, provide a link to the Creative Commons license, and indicate if changes were made. 
tive of Leray-Schauder theorem and Krasnoselskii's fixed point theorem in a cone, Bai and Fang in [4] obtained the existence of positive solutions for the following singular coupled system of nonlinear fractional differential equations:

$$
\begin{cases}D^{s} u=f(t, v), & 0<t<1, \\ D^{p} v=g(t, u), & 0<t<1,\end{cases}
$$

where $0<s<1,0<p<1, D^{s}, D^{p}$ are two standard Riemann-Liouville fractional derivatives, $f, g:(0,1] \times[0,+\infty) \rightarrow[0,+\infty)$ are two given continuous functions. Su [5] established sufficient conditions for the existence of solutions for the following coupled system of fractional differential equations with two-point boundary conditions:

$$
\left\{\begin{array}{l}
D^{\alpha} u(t)=f\left(t, v(t), D^{\mu} v(t)\right), \quad 0<t<1, \\
D^{\beta} v(t)=g\left(t, u(t), D^{\nu} u(t)\right), \quad 0<t<1, \\
u(0)=u(1)=v(0)=v(1)=0,
\end{array}\right.
$$

where $1<\alpha, \beta<2, \mu, v>0, \alpha-v \geq 1, \beta-\mu \geq 1, f, g:[0,1] \times R \times R \rightarrow R$ are given functions, and $D$ is the standard Riemann-Liouville fractional derivative. Ahmad and Nieto [6] extended the results of [5] to a three-point boundary value problem for the following coupled system of fractional differential equations:

$$
\begin{cases}D^{\alpha} u(t)=f\left(t, v(t), D^{\mu} v(t)\right), & 0<t<1, \\ D^{\beta} v(t)=g\left(t, u(t), D^{v} u(t)\right), & 0<t<1, \\ u(0)=0, & u(1)=\gamma u(\eta),\end{cases}
$$

where $1<\alpha, \beta<2, \mu, v, \gamma>0, \alpha-v \geq 1, \beta-\mu \geq 1,0<\eta<1, \gamma \eta^{\alpha-1}<1, \gamma \eta^{\beta-1}<1 f, g$ : $[0,1] \times R \times R \rightarrow R$ are given functions, and $D$ is the standard Riemann-Liouville fractional derivative. Yang [7] established sufficient conditions for the existence and nonexistence of positive solutions to boundary values problem for a coupled system of nonlinear fractional differential equations as follows:

$$
\left\{\begin{array}{l}
D^{\alpha} u(t)+a(t) f(t, v(t))=0, \quad 0<t<1, \\
D^{\beta} v(t)+b(t) g(t, u(t))=0, \quad 0<t<1, \\
u(0)=0, \quad u(1)=\int_{0}^{1} \phi(t) u(t) d t, \\
v(0)=0, \quad v(1)=\int_{0}^{1} \psi(t) v(t) d t,
\end{array}\right.
$$

where $1<\alpha, \beta \leq 2, a, b \in C((0,1),[0,+\infty)), \phi, \psi \in L^{1}[0,1]$ are nonnegative and $f, g \in$ $C([0,1] \times[0,+\infty),[0,+\infty))$, and $D$ is the standard Riemann-Liouville fractional derivative.

Inspired by the above papers and some known results on fractional differential equations with integral boundary conditions [8-30], this paper is to establish the existence and uniqueness of nontrivial solutions to FBVP (1.1) under the conditions that the nonlinear terms $f, g$ of FBVP (1.1) are allowed to be sign-changing and unbounded from below. Finally, it is worth mentioning that the main technique used here is the topological 
degree theory, the theory of linear operators. As far as we know, there are few works that deal with system of fractional differential equations with Riemann-Stieltjes integral conditions where the nonlinear terms may be unbounded from below. The main results here are different from [4-32, 35-37].

\section{Preliminaries and lemmas}

Let $E=C[0,1]$ be a Banach space with the norm $\|u\|=\max _{0 \leq t \leq 1}|u(t)|$ for $u \in E$. Let $P=$ $\{u \in E \mid u(t) \geq 0, t \in[0,1]\}$. Then $P$ is a total cone in $E$, that is, $E=\overline{P-P}$. Let $P^{*}=\left\{g \in E^{*} \mid\right.$ $g(u) \geq 0$ for all $u \in P$ \}. Then $P^{*}$ is the dual cone of $P$. Let $E^{*}$ denote the dual space of $E$, then by Riesz representation theorem, $E^{*}$ is given by

$$
\begin{aligned}
E^{*}= & \{v \mid v \text { is right continuous on }[0,1) \text { and is bounded variation on } \\
& {[0,1] \text { with } v(0)=0\} . }
\end{aligned}
$$

Let $E^{2}$ be equipped with the norm $\|(u, v)\|_{1}=\|u\|+\|v\|$. Then $E^{2}$ is also a real Banach space and $P^{2}=P \times P$ is a cone in $E^{2}$. Let $\left(u_{1}, v_{1}\right) \geq\left(u_{2}, v_{2}\right)$ denote $u_{1} \geq u_{2}, v_{1} \geq v_{2}$ for $\left(u_{1}, v_{1}\right),\left(u_{2}, v_{2}\right) \in E^{2}$ and $B_{r}=\left\{(u, v) \in E^{2} \mid\|(u, v)\|_{1}<r\right\}$ for any $r>0$.

Definition 2.1 The Riemann-Liouville fractional integral of order $\alpha>0$ of a function $y:(0,+\infty) \rightarrow \mathbb{R}$ is given by

$$
I_{0+}^{\alpha} y(t)=\frac{1}{\Gamma(\alpha)} \int_{0}^{t}(t-s)^{\alpha-1} y(s) d s
$$

provided the right-hand side is defined on $(0,+\infty)$ pointwisely.

Definition 2.2 The Riemann-Liouville fractional derivative of order $\alpha>0$ of a function $y:(0,+\infty) \rightarrow \mathbb{R}$ is given by

$$
D_{0+}^{\alpha} y(t)=\frac{1}{\Gamma(n-\alpha)}\left(\frac{d}{d t}\right)^{n} \int_{0}^{t} \frac{y(s)}{(t-s)^{\alpha-n+1}} d s,
$$

where $n=[\alpha]+1,[\alpha]$ denotes the integer part of the number $\alpha$, provided that the righthand side is defined on $(0,+\infty)$ pointwisely.

Lemma 2.1 Let $\alpha>0$. If we assume $u \in C(0,1) \cap L(0,1)$, then the fractional differential equation

$$
D_{0+}^{\alpha} u(t)=0
$$

has $u(t)=c_{1} t^{\alpha-1}+c_{2} t^{\alpha-2}+\cdots+c_{N} t^{\alpha-N}, c_{i} \in \mathbb{R}, i=1,2, \ldots, N$, as unique solutions, where $N$ is the smallest integer greater than or equal to $\alpha$.

Lemma 2.2 Assume that $u \in C(0,1) \cap L(0,1)$ with a fractional derivative of order $\alpha>0$ that belongs to $C(0,1) \cap L(0,1)$. Then

$$
I_{0+}^{\alpha} D_{0+}^{\alpha} u(t)=u(t)+c_{1} t^{\alpha-1}+c_{2} t^{\alpha-2}+\cdots+c_{N} t^{\alpha-N}
$$

for some $c_{i} \in \mathbb{R}, i=1,2, \ldots, N, N$ is the smallest integer greater than or equal to $\alpha$. 
Lemma 2.3 ([31]) Given $y \in L(0,1)$ and $2<\alpha \leq 3$, then the unique solution of

$$
\left\{\begin{array}{l}
D_{0+}^{\alpha} u(t)+y(t)=0, \quad 0<t<1, \\
u(0)=u^{\prime}(0)=0, \quad u(1)=0
\end{array}\right.
$$

is $u(t)=\int_{0}^{1} G_{0}(t, s) y(s) d s$, where

$$
G_{0}(t, s)= \begin{cases}\frac{[t(1-s)]^{\alpha-1}-(t-s)^{\alpha-1}}{\Gamma(\alpha)}, & 0 \leq s \leq t \leq 1, \\ \frac{[t(1-s)]^{\alpha-1}}{\Gamma(\alpha)}, & 0 \leq t \leq s \leq 1 .\end{cases}
$$

Lemma 2.4 ([31]) The Green function $G_{0}(t, s)$ has the following properties:

(1) $\Gamma(\alpha) k(t) q(s) \leq G_{0}(t, s) \leq(\alpha-1) q(s)$ for $t, s \in[0,1]$,

(2) $\Gamma(\alpha) k(t) q(s) \leq G_{0}(t, s) \leq(\alpha-1) k(t)$ for $t, s \in[0,1]$,

where

$$
k(t)=\frac{t^{\alpha-1}(1-t)}{\Gamma(\alpha)}, \quad q(s)=\frac{s(1-s)^{\alpha-1}}{\Gamma(\alpha)} .
$$

By Lemma 2.1, the unique solution of the problem

$$
\left\{\begin{array}{l}
D_{0+}^{\alpha} u(t)=0, \quad 0<t<1 \\
u(0)=u^{\prime}(0)=0, \quad u(1)=1
\end{array}\right.
$$

is $u(t)=t^{\alpha-1}$. Then it is easy to verify, as a consequence of Lemma 2.3, that FBVP (1.1) is equivalent to the system of perturbed integral equations

$$
\left\{\begin{array}{l}
u(t)=\int_{0}^{1} G_{0}(t, s) h(s) f(s, v(s)) d s+t^{\alpha-1} \int_{0}^{1} u(\tau) d \beta(\tau), \\
v(t)=\int_{0}^{1} G_{0}(t, s) h(s) g(s, u(s)) d s+t^{\alpha-1} \int_{0}^{1} v(\tau) d \beta(\tau) .
\end{array}\right.
$$

Define $\Gamma=\int_{0}^{1} t^{\alpha-1} d \beta(t), g_{\beta}(s)=\int_{0}^{1} G_{0}(t, s) d \beta(t)$. Then we have the following lemma.

Lemma 2.5 Given $y(t) \in C(0,1) \cap L(0,1)$ and $2<\alpha \leq 3$, then

$$
\begin{cases}D_{0+}^{\alpha} u(t)+y(t)=0, & 0<t<1, \\ u(0)=u^{\prime}(0)=0, & u(1)=\int_{0}^{1} u(\tau) d \beta(\tau),\end{cases}
$$

has the unique solution

$$
u(t)=\int_{0}^{1} G(t, s) y(s) d s
$$

where the Green function $G(t, s)$ is given by

$$
G(t, s)=\frac{t^{\alpha-1}}{1-\Gamma} g_{\beta}(s)+G_{0}(t, s), \quad t, s \in[0,1]
$$


Proof Multiply (2.1) by $d \beta(t)$ on both sides and integrate over $[0,1]$ to obtain

$$
\begin{aligned}
\int_{0}^{1} u(t) d \beta(t) & =\int_{0}^{1} \int_{0}^{1} G_{0}(t, s) y(s) d s d \beta(t)+\int_{0}^{1} t^{\alpha-1} \int_{0}^{1} u(\tau) d \beta(\tau) d \beta(t) \\
& =\int_{0}^{1} \int_{0}^{1} G_{0}(t, s) y(s) d s d \beta(t)+\int_{0}^{1} t^{\alpha-1} d \beta(t) \int_{0}^{1} u(\tau) d \beta(\tau) .
\end{aligned}
$$

Consequently,

$$
\int_{0}^{1} u(t) d \beta(t)=\frac{1}{1-\Gamma} \int_{0}^{1} \int_{0}^{1} G_{0}(t, s) y(s) d s d \beta(t) .
$$

Replacing $\int_{0}^{1} u(\tau) d \beta(\tau)$ in (2.1) with the above equality, we obtain

$$
\begin{aligned}
u(t) & =\int_{0}^{1} G_{0}(t, s) y(s) d s+\frac{t^{\alpha-1}}{1-\Gamma} \int_{0}^{1}\left(\int_{0}^{1} G_{0}(t, s) d \beta(t)\right) y(s) d s \\
& =\int_{0}^{1}\left(G_{0}(t, s)+\frac{t^{\alpha-1}}{1-\Gamma} g_{\beta}(s)\right) y(s) d s \\
& =\int_{0}^{1} G(t, s) y(s) d s .
\end{aligned}
$$

Reversely, if $u(t)=\int_{0}^{1} G(t, s) y(s) d s$, then $u(0)=0$ and $u(1)=\int_{0}^{1} u(\tau) d \beta(\tau)$ via (2.1). According to Definition 2.2, Lemma 2.3, and Lemma 2.4, $D_{0_{+}}^{\alpha} u(t)+y(t)=0$ holds.

By Lemma 2.5, $(u, v) \in E^{2}$ is a solution of FBVP (1.1) if and only if

$$
\left\{\begin{array}{l}
u(t)=\int_{0}^{1} G(t, s) h(s) f(s, v(s)) d s, \\
v(t)=\int_{0}^{1} G(t, s) h(s) g(s, u(s)) d s .
\end{array}\right.
$$

Define

$$
\begin{aligned}
& \left(A_{1} v\right)(t)=\int_{0}^{1} G(t, s) h(s) f(s, v(s)) d s \\
& \left(A_{2} u\right)(t)=\int_{0}^{1} G(t, s) h(s) g(s, u(s)) d s \\
& A(u, v)(t)=\left(\left(A_{1} v\right)(t),\left(A_{2} u\right)(t)\right) .
\end{aligned}
$$

It is easy to show that $A: E^{2} \rightarrow E^{2}$ is a completely continuous nonlinear operator, and if $(u, v) \in E^{2}$ is a fixed point of $A$, then $(u, v)$ is a solution of FBVP (1.1) by Lemma 2.5.

For any $u \in E$, define $K: E \rightarrow E$ as follows:

$$
(K u)(t)=\int_{0}^{1} G(t, s) h(s) u(s) d s, \quad u \in E .
$$

Then $K: E \rightarrow E$ is a completely continuous linear operator and $K(P) \subset P$ holds. Since $h \in C(0,1) \cap L(0,1)$ with $\int_{0}^{1} h(t) d t>0$, by [32], the spectral radius $r(K)$ of $K$ is positive. 
The Krein-Rutman theorem [33] asserts that there exist $\phi \in P \backslash\{0\}$ and $\omega \in P^{*} \backslash\{0\}$ corresponding to the number $\lambda_{1}=1 / r(K)$ relative to $K$ such that

$$
\lambda_{1} K \phi=\phi
$$

and

$$
\lambda_{1} K^{*} \omega=\omega, \quad \omega(1)=1 .
$$

Here $K^{*}: E^{*} \rightarrow E^{*}$ is the dual operator of $K$ given by

$$
\left(K^{*} v\right)(s)=\int_{0}^{s} \int_{0}^{1} G(t, \tau) h(\tau) d v(t) d \tau, \quad v \in E^{*} .
$$

Now we testify that $K^{*}: E^{*} \rightarrow E^{*}$ is the dual operator of $K$. In fact,

$$
\begin{aligned}
\left\langle K^{*} v(s), u(s)\right\rangle & =\int_{0}^{1} u(s) d K^{*} v(s)=\int_{0}^{1} u(s) \int_{0}^{1} G(t, s) h(s) d v(t) d s \\
& =\int_{0}^{1}\left(\int_{0}^{1} G(t, s) h(s) u(s) d s\right) d v(t) \\
& =\int_{0}^{1}(K u)(t) d v(t)=\langle v(t),(K u)(t)\rangle .
\end{aligned}
$$

So $K^{*}: E^{*} \rightarrow E^{*}$ is the dual operator of $K$.

The continuity of $G$, the integrability of $h$, and the representation of $K^{*}$ induce that $\omega \in C^{1}[0,1]$. Let $e(t):=\omega^{\prime}(t)$. Then $e \in P \backslash\{0\}$, and (2.5) can be rewritten equivalently as

$$
r(K) e(s)=\int_{0}^{1} G(t, s) h(s) e(t) d t, \quad \int_{0}^{1} e(t) d t=1 .
$$

Lemma 2.6 Let $0 \leq \Gamma=\int_{0}^{1} t^{\alpha-1} d \beta(t)<1$ and $g_{\beta}(s)=\int_{0}^{1} G_{0}(t, s) d \beta(t) \geq 0$ for $s \in[0,1]$, then there exists $\delta>0$ such that $P_{0}=\left\{u \in P \mid \int_{0}^{1} u(t) e(t) d t \geq \delta\|u\|\right\}$ is a subcone of $P$ and $K(P) \subset P_{0}$.

Proof Let $\delta=\int_{0}^{1} \frac{(1-t) t^{\alpha-1}}{\alpha-1} e(t) d t$. It is obvious that $G(t, s)>0$ holds for $t, s \in(0,1)$. By Lemma 2.4 and (2.2),

$$
G(t, s)=G_{0}(t, s)+\frac{t^{\alpha-1}}{1-\Gamma} g_{\beta}(s) \leq G_{0}(t, s)+\frac{1}{1-\Gamma} g_{\beta}(s) \leq \frac{s(1-s)^{\alpha-1}}{\Gamma(\alpha-1)}+\frac{g_{\beta}(s)}{1-\Gamma}
$$

and

$$
\begin{aligned}
G(t, s) & =G_{0}(t, s)+\frac{t^{\alpha-1}}{1-\Gamma} g_{\beta}(s) \geq \frac{(1-t) s[t(1-s)]^{\alpha-1}}{(\alpha-1) \Gamma(\alpha-1)}+\frac{(1-t) t^{\alpha-1} g_{\beta}(s)}{(\alpha-1)(1-\Gamma)} \\
& =\frac{(1-t) t^{\alpha-1}}{\alpha-1}\left(\frac{s(1-s)^{\alpha-1}}{\Gamma(\alpha-1)}+\frac{g_{\beta}(s)}{1-\Gamma}\right) .
\end{aligned}
$$

For any $u \in P$,

$$
\max _{t \in[0,1]}|(K u)(t)|=\max _{t \in[0,1]}\left|\int_{0}^{1} G(t, s) h(s) u(s) d s\right| \leq \int_{0}^{1}\left(\frac{s(1-s)^{\alpha-1}}{\Gamma(\alpha-1)}+\frac{g_{\beta}(s)}{1-\Gamma}\right) h(s) u(s) d s .
$$


Then we have

$$
\begin{aligned}
\int_{0}^{1}(K u)(t) e(t) d t & =\int_{0}^{1} \int_{0}^{1} G(t, s) h(s) u(s) d s e(t) d t \\
& \geq \int_{0}^{1} \int_{0}^{1} \frac{(1-t) t^{\alpha-1}}{\alpha-1}\left(\frac{s(1-s)^{\alpha-1}}{\Gamma(\alpha-1)}+\frac{g_{\beta}(s)}{1-\Gamma}\right) h(s) u(s) d s e(t) d t \\
& =\int_{0}^{1} \frac{(1-t) t^{\alpha-1}}{\alpha-1} e(t) d t \cdot \int_{0}^{1}\left(\frac{s(1-s)^{\alpha-1}}{\Gamma(\alpha-1)}+\frac{g_{\beta}(s)}{1-\Gamma}\right) h(s) u(s) d s \\
& \geq \int_{0}^{1} \frac{(1-t) t^{\alpha-1}}{\alpha-1} e(t) d t \cdot\|K u\| \\
& =\delta\|K u\| .
\end{aligned}
$$

That means $K(P) \subset P_{0}$.

Lemma 2.7 ([34]) Let E be a real Banach space and $\Omega \subset E$ be a bounded open set with $0 \in \Omega$. Suppose that $A: \bar{\Omega} \rightarrow E$ is a completely continuous operator. (1) If there is $y_{0} \in E$ with $y_{0} \neq 0$ such that $u \neq A u+\mu y_{0}$, for all $u \in \partial \Omega$ and $\mu \geq 0$, then $\operatorname{deg}(I-A, \Omega, 0)=0$. (2) If $A u \neq \mu u$ for all $u \in \partial \Omega$ and $\mu \geq 1$, then $\operatorname{deg}(I-A, \Omega, 0)=1$. Here deg stands for the Leray-Schauder topological degree in $E$.

Lemma 2.8 Assume that the following assumptions are satisfied:

$\left(C_{1}\right)$ There exist $\phi \in P \backslash\{0\}, \omega \in P^{*} \backslash\{0\}$ such that (2.4), (2.5) hold and $K$ maps $P$ into $P_{0}$.

$\left(C_{2}\right)$ There exists a continuous operator $H: E \rightarrow P$ such that

$$
\lim _{\|u\|+\|v\| \rightarrow+\infty} \frac{\|H u\|+\|H v\|}{\|u\|+\|v\|}=0 .
$$

$\left(C_{3}\right)$ There exist two bounded continuous operators $F, G: E \rightarrow E$ and $u_{0}, v_{0} \in E$ such that $\left(F v+v_{0}+H v, G u+u_{0}+H u\right) \in P^{2}$ for all $(u, v) \in E^{2}$.

$\left(C_{4}\right)$ There exist $m_{0}, n_{0} \in E$ and $\zeta>0$ such that $(K F v, K G u) \geq\left(\lambda_{1}(1+\zeta) K v-K H v-\right.$ $\left.m_{0}, \lambda_{1}(1+\zeta) K u-K H u-n_{0}\right)$ for all $(u, v) \in E^{2}$.

Let $A_{1}=K F, A_{2}=K G, A(u, v)(t)=\left(\left(A_{1} v\right)(t),\left(A_{2} u\right)(t)\right)$, then there exists $R>0$ such that

$$
\operatorname{deg}\left(I-A, B_{R}, 0\right)=0,
$$

where $B_{r}=\left\{(u, v) \in E^{2} \mid\|(u, v)\|_{1}<r\right\}$ for any $r>0$.

Proof Choose a constant $l_{0}=\left(\delta \lambda_{1}\right)^{-1}\left(1+\zeta^{-1}\right)+\|K\|>0$. By $\left(C_{2}\right)$, for $0<\varepsilon_{0}<l_{0}^{-1}$, there exists $R_{1}>0$ such that $\|u\|+\|v\|>R_{1}$ implies

$$
\|H u\|+\|H v\|<\varepsilon_{0}(\|u\|+\|v\|)
$$

Now we shall show

$$
(u, v) \neq A(u, v)+\mu(\phi, \phi) \quad \text { for any }(u, v) \in \partial B_{R} \text { and } \mu \geq 0 \text {, }
$$

provided that $R$ is sufficiently large. 
Lu et al. Advances in Difference Equations (2018) 2018:306

Page 8 of 15

In fact, if (2.8) is not true, then there exist $\left(u_{1}, v_{1}\right) \in \partial B_{R}$ and $\mu_{1} \geq 0$ satisfying

$$
(u, v)=A(u, v)+\mu(\phi, \phi),
$$

that is,

$$
\left(u_{1}, v_{1}\right)=\left(K F v_{1}+\mu_{1} \phi, K G u_{1}+\mu_{1} \phi\right)
$$

Since $\phi \in P \backslash\{0\}, e(t) \in P \backslash\{0\}, \int_{0}^{1} \phi(t) e(t) d t>0$. Multiply (2.10) by $e(t)$ on both sides and integrate respectively on $[0,1]$. Then by $\left(C_{4}\right),(2.6)$, we get

$$
\begin{aligned}
\int_{0}^{1} u_{1}(t) e(t) d t= & \int_{0}^{1}\left(K F v_{1}\right)(t) e(t) d t+\mu_{1} \int_{0}^{1} \phi(t) e(t) d t \\
\geq & \lambda_{1}(1+\zeta) \int_{0}^{1} \int_{0}^{1} G(t, s) h(s) v_{1}(s) d s e(t) d t \\
& -\int_{0}^{1}\left(K H v_{1}\right)(t) e(t) d t-\int_{0}^{1} m_{0}(t) e(t) d t \\
= & \lambda_{1}(1+\zeta) \int_{0}^{1} \int_{0}^{1} G(t, s) h(s) v_{1}(s) e(t) d s d t \\
& -\int_{0}^{1} \int_{0}^{1} G(t, s) h(s)\left(H v_{1}\right)(s) e(t) d s d t-\int_{0}^{1} m_{0}(t) e(t) d t \\
= & \lambda_{1}(1+\zeta) \int_{0}^{1}\left[\int_{0}^{1} G(t, s) h(s) e(t) d t\right] v_{1}(s) d s \\
& -\int_{0}^{1}\left[\int_{0}^{1} G(t, s) h(s) e(t) d t\right]\left(H v_{1}\right)(s) d s-\int_{0}^{1} m_{0}(t) e(t) d t \\
= & \lambda_{1}(1+\zeta) r(K) \int_{0}^{1} e(s) v_{1}(s) d s \\
& -r(K) \int_{0}^{1}\left(H v_{1}\right)(t) e(t) d t-\int_{0}^{1} m_{0}(t) e(t) d t \\
= & (1+\zeta) \int_{0}^{1}\left(H v_{1}\right)(s) e(s) d s-\int_{0}^{1} m_{0}(t) e(t) d t \\
& r(t) d t \\
& \\
& \\
&
\end{aligned}
$$

and

$$
\begin{aligned}
\int_{0}^{1} v_{1}(t) e(t) d t & =\int_{0}^{1}\left(K G u_{1}\right)(t) e(t) d t+\mu_{1} \int_{0}^{1} \phi(t) e(t) d t \\
& \geq(1+\zeta) \int_{0}^{1} u_{1}(t) e(t) d t-r(K) \int_{0}^{1}\left(H u_{1}\right)(t) e(t) d t-\int_{0}^{1} n_{0}(t) e(t) d t
\end{aligned}
$$


According to the two above inequalities, we obtain

$$
\begin{aligned}
\int_{0}^{1}\left[u_{1}(t)+v_{1}(t)\right] e(t) d t \geq & (1+\zeta) \int_{0}^{1}\left[u_{1}(t)+v_{1}(t)\right] e(t) d t \\
& -r(K) \int_{0}^{1}\left[\left(H u_{1}\right)(t)+\left(H v_{1}\right)(t)\right] e(t) d t \\
& -\int_{0}^{1}\left[m_{0}(t)+n_{0}(t)\right] e(t) d t
\end{aligned}
$$

Then we derive

$$
\begin{aligned}
\int_{0}^{1}\left[u_{1}(t)+v_{1}(t)\right] e(t) d t \leq & \zeta^{-1}\left[r(K) \int_{0}^{1}\left[\left(H u_{1}\right)(t)+\left(H v_{1}\right)(t)\right] e(t) d t\right. \\
& \left.+\int_{0}^{1}\left[m_{0}(t)+n_{0}(t)\right] e(t) d t\right]
\end{aligned}
$$

By computation, we obtain

$$
\begin{aligned}
& \int_{0}^{1}\left(K H u_{1}\right)(t) e(t) d t=r(K) \int_{0}^{1}\left(H u_{1}\right)(t) e(t) d t \\
& \int_{0}^{1}\left(K H v_{1}\right)(t) e(t) d t=r(K) \int_{0}^{1}\left(H v_{1}\right)(t) e(t) d t
\end{aligned}
$$

By (2.6), (2.7), (2.11), and (2.12), we get

$$
\begin{aligned}
\int_{0}^{1}[ & \left.u_{1}(t)+v_{1}(t)+\left(K H v_{1}\right)(t)+\left(K H u_{1}\right)(t)+\left(K u_{0}\right)(t)+\left(K v_{0}\right)(t)\right] e(t) d t \\
\leq & \zeta^{-1}\left[r(K) \int_{0}^{1}\left[\left(H v_{1}\right)(t)+\left(H u_{1}\right)(t)\right] e(t) d t+\int_{0}^{1}\left[m_{0}(t)+n_{0}(t)\right] e(t) d t\right] \\
& +r(K) \int_{0}^{1}\left(H u_{1}\right)(t) e(t) d t+r(K) \int_{0}^{1}\left(H v_{1}\right)(t) e(t) d t \\
& +\int_{0}^{1}\left(K u_{0}\right)(t) e(t) d t+\int_{0}^{1}\left(K u_{0}\right)(t) e(t) d t \\
= & \zeta^{-1}(1+\zeta) r(K) \int_{0}^{1}\left[\left(H u_{1}\right)(t)+\left(H v_{1}\right)(t)\right] e(t) d t \\
& +\zeta^{-1} \int_{0}^{1}\left[m_{0}(t)+n_{0}(t)\right] e(t) d t+\int_{0}^{1}\left[\left(K u_{0}\right)(t)+\left(K v_{0}\right)(t)\right] e(t) d t \\
\leq & \zeta^{-1}(1+\zeta) r(K)(\|H u\|+\|H v\|)+\zeta^{-1} \int_{0}^{1}\left[m_{0}(t)+n_{0}(t)\right] e(t) d t \\
& +\int_{0}^{1}\left[\left(K u_{0}\right)(t)+\left(K v_{0}\right)(t)\right] e(t) d t \\
\leq & \zeta^{-1}(1+\zeta) r(K) \varepsilon_{0}(\|u\|+\|v\|)+l_{1},
\end{aligned}
$$

where $l_{1}=\zeta^{-1} \int_{0}^{1}\left[m_{0}(t)+n_{0}(t)\right] e(t) d t+\int_{0}^{1}\left[\left(K u_{0}\right)(t)+\left(K v_{0}\right)(t)\right] e(t) d t$ is a constant. 
$\left(C_{3}\right)$ shows $\left(F v_{1}+v_{0}+H v_{1}, G u_{1}+u_{0}+H u_{1}\right) \in P^{2}$ and $\left(C_{1}\right)$ implies $\mu_{1} \phi=\mu_{1} \lambda_{1} K \phi \in P_{0}$. Then $\left(C_{1}\right),(2.10)$, and Lemma 2.6 tell us that

$$
\begin{aligned}
& u_{1}+K H v_{1}+K v_{0}=K F v_{1}+\mu_{1} \phi+K H v_{1}+K v_{0}=K\left(F v_{1}+H v_{1}+v_{0}\right)+\mu_{1} \phi \in P_{0}, \\
& v_{1}+K H u_{1}+K u_{0}=K G u_{1}+\mu_{1} \phi+K H u_{1}+K u_{0}=K\left(G v_{1}+H u_{1}+u_{0}\right)+\mu_{1} \phi \in P_{0} .
\end{aligned}
$$

The definition of $P_{0}$ yields

$$
\begin{aligned}
& \int_{0}^{1}\left(u_{1}+K H v_{1}+K v_{0}\right)(t) e(t) d t \geq \delta\left\|u_{1}+K H v_{1}+K v_{0}\right\| \geq \delta\left\|u_{1}\right\|-\delta\left\|K H v_{1}\right\|-\delta\left\|K v_{0}\right\| \\
& \int_{0}^{1}\left(v_{1}+K H u_{1}+K u_{0}\right)(t) e(t) d t \geq \delta\left\|v_{1}+K H u_{1}+K u_{0}\right\| \geq \delta\left\|v_{1}\right\|-\delta\left\|K H u_{1}\right\|-\delta\left\|K u_{0}\right\|
\end{aligned}
$$

where $\delta$ is given in Lemma 2.6. By adding the above two inequalities, we obtain

$$
\begin{aligned}
& \int_{0}^{1}\left(u_{1}+v_{1}+K H u_{1}+K H v_{1}+K u_{0}+K v_{0}\right)(t) e(t) d t \\
& \quad \geq \delta\left(\left\|v_{1}\right\|+\|u\|\right)-\delta\left(\left\|K H u_{1}\right\|+\left\|K H v_{1}\right\|\right)-\delta\left(\left\|K u_{0}\right\|+\left\|K v_{0}\right\|\right)
\end{aligned}
$$

It follows from (2.7), (2.13), and (2.14) that

$$
\begin{aligned}
\left\|u_{1}\right\|+\left\|v_{1}\right\| \leq & \delta^{-1} \int_{0}^{1}\left(u_{1}+v_{1}+K H u_{1}+K H v_{1}+K u_{0}+K v_{0}\right)(t) e(t) d t \\
& +\left\|K H u_{1}\right\|+\left\|K H v_{1}\right\|+\left\|K u_{0}\right\|+\left\|K v_{0}\right\| \\
\leq & \varepsilon_{0}\left(\delta \lambda_{1}\right)^{-1}\left(1+\zeta^{-1}\right)\left(\left\|u_{1}\right\|+\left\|v_{1}\right\|\right)+l_{1} \delta^{-1} \\
& +\varepsilon_{0}\|K\| \cdot\left(\left\|u_{1}\right\|+\left\|v_{1}\right\|\right)+\left\|K u_{0}\right\|+\left\|K v_{0}\right\| \\
= & \varepsilon_{0} l_{0}\left(\left\|u_{1}\right\|+\left\|v_{1}\right\|\right)+l_{2}
\end{aligned}
$$

where $l_{2}=l_{1} \delta^{-1}+\left\|K u_{0}\right\|+\left\|K v_{0}\right\|$ is a constant.

Since $0<\varepsilon_{0} l_{0}<1$, then (2.15) deduces that (2.8) holds provided that $R$ is sufficiently large such that $R>\max \left\{l_{2} /\left(1-\varepsilon_{0} l_{0}\right), R_{1}\right\}$. By $(2.15)$ and Lemma 2.7 , we have

$\operatorname{deg}\left(I-A, B_{R}, 0\right)=0$.

\section{Existence}

Theorem 3.1 Assume that the following conditions are satisfied:

$\left(\mathrm{A}_{1}\right) f, g:[0,1] \times \mathbb{R} \rightarrow \mathbb{R}$ are continuous.

$\left(\mathrm{A}_{2}\right)$ There exist nonnegative functions $b_{i}(t), c_{i}(t) \in C[0,1]$ with $c_{i}(t) \not \equiv$ and two continuous even functions $B_{i}: \mathbb{R} \rightarrow \mathbb{R}^{+}$such that

$$
\begin{array}{ll}
f(t, x) \geq-b_{1}(t)-c_{1}(t) B_{1}(x) & \text { for all } x \in \mathbb{R}, \\
g(t, y) \geq-b_{2}(t)-c_{2}(t) B_{2}(y) & \text { for all } y \in \mathbb{R} .
\end{array}
$$

Moreover, $B_{i}$ is nondecreasing on $\mathbb{R}^{+}$and satisfies $\lim _{x \rightarrow+\infty} \frac{B_{i}(x)}{x}=0,(i=1,2)$. 
(A $\left.\mathrm{A}_{3}\right) \liminf _{x \rightarrow+\infty} \frac{f(t, x)}{x}>\lambda_{1}, \liminf _{x \rightarrow+\infty} \frac{g(t, y)}{y}>\lambda_{1}$, uniformly on $t \in[0,1]$.

(A $\left.\mathrm{A}_{4}\right) \lim \sup _{x \rightarrow 0}\left|\frac{f(t, x)}{x}\right|<\lambda_{1}$, lim sup $\operatorname{su}_{x \rightarrow 0}\left|\frac{g(t, y)}{y}\right|<\lambda_{1}$, uniformly on $t \in[0,1]$.

Here $\lambda_{1}=1 / r(K)$ is a number and the operator $K$ is defined by (2.3).

Then FBVP (1.1) has at least one nontrivial solution.

Proof We first show that all conditions in Lemma 2.8 are satisfied. By Lemma 2.6, condition $\left(\mathrm{C}_{1}\right)$ of Lemma 2.8 is satisfied. $\left(T_{i} u\right)(t)=B_{i}(u(t))(i=1,2)$ for any $u \in E$. Obviously $T_{1}, T_{2}: E \rightarrow P$ are continuous operators. By $\left(\mathrm{A}_{2}\right)$, for any $\varepsilon>0$, there is $L>0$ such that when $z>L, B_{i}(z)<\varepsilon z$ holds. Thus, for $w \in E$ with $\|w\|>L, B_{i}(\|w\|)<\varepsilon\|w\|$ holds. The fact that $B_{i}$ is nondecreasing on $\mathbb{R}^{+}$yields $\left(T_{i} w\right)(t) \leq T_{i}(\|w\|)$ for any $w \in P, t \in[0,1]$. Since $B_{i}: \mathbb{R} \rightarrow \mathbb{R}^{+}$is an even function, $\left\|T_{i} w\right\| \leq T_{i}(\|w\|)$ holds for $w \in E$. Therefore,

$$
\left\|T_{i} w\right\| \leq T_{i}(\|w\|)<\varepsilon\|w\|, \quad \forall w \in E \text { with }\|w\|>L,
$$

that is, $\lim _{\|w\| \rightarrow+\infty} \frac{\left\|T_{i} w\right\|}{\|w\|}=0$.

Define $(H w)(t)=\max \left\{C_{1}\left(T_{1} w\right)(t), C_{2}\left(T_{2} w\right)(t)\right\}$ for any $w \in E, t \in[0,1]$, where $C_{i}=$ $\max _{t \in[0,1]} c_{i}(t), i=1,2 . \operatorname{By}_{\| \lim _{\|w\| \rightarrow+\infty}} \frac{\left\|T_{i} w\right\|}{\|w\|}=0, \lim _{\|u\|+\|v\| \rightarrow+\infty} \frac{\left\|T_{i} u\right\|+\left\|T_{i} v\right\|}{\|u\|+\|v\|}=0$ holds. Therefore $\lim _{\|u\|+\|v\| \rightarrow+\infty} \frac{\|H u\|+\|H v\|}{\|u\|+\|v\|}=0$ holds. Then we obtain that $H$ satisfies condition $\left(\mathrm{C}_{2}\right)$ in Lemma 2.8 .

Take $v_{0}(t) \equiv b_{1}=\max _{t \in[0,1]} b_{1}(t)>0, u_{0}(t) \equiv b_{2}=\max _{t \in[0,1]} b_{2}(t)>0$, and $(F v)(t)=$ $f(t, v(t)),(G u)(t)=g(t, u(t))$ for $t \in[0,1],(u, v) \in E^{2}$, then it follows from $\left(\mathrm{A}_{1}\right)$ that

$$
\left(F v+v_{0}+H v, G u+u_{0}+H u\right) \in P^{2} \quad \text { for all }(u, v) \in E^{2},
$$

which shows that condition $\left(\mathrm{C}_{3}\right)$ in Lemma 2.8 holds.

By $\left(\mathrm{A}_{3}\right)$, there exist $\varepsilon_{1}>0$ and a sufficiently large number $L_{1}>0$ such that

$$
f(t, x) \geq \lambda_{1}\left(1+\varepsilon_{1}\right) x, \quad g(t, y) \geq \lambda_{1}\left(1+\varepsilon_{1}\right) y, \quad \forall x, y \geq L_{1} .
$$

Combining (3.1) with $\left(\mathrm{A}_{2}\right)$, the above constants $b_{1}, b_{2}$ satisfy

$$
\begin{aligned}
& f(t, x) \geq \lambda_{1}\left(1+\varepsilon_{1}\right) x-b_{1}-c_{1} B_{1}(x), \\
& g(t, y) \geq \lambda_{1}\left(1+\varepsilon_{1}\right) y-b_{2}-c_{2} B_{1}(y) \quad \text { for all } x, y \in \mathbb{R},
\end{aligned}
$$

and so

$$
(F v, G u) \geq\left(\lambda_{1}\left(1+\varepsilon_{1}\right) v-b_{1}-H v, \lambda_{1}\left(1+\varepsilon_{1}\right) u-b_{2}-H u\right) \quad \text { for all }(u, v) \in E^{2} .
$$

Since $K$ is a positive linear operator, from (3.2), we have

$$
\begin{aligned}
((K F v)(t),(K G u)(t)) \geq & \left(\lambda_{1}\left(1+\varepsilon_{1}\right)(K v)(t)-K b_{1}-(K H v)(t),\right. \\
& \left.\lambda_{1}\left(1+\varepsilon_{1}\right)(K u)(t)-K b_{2}-(K H u)(t)\right) \quad \forall t \in[0,1],(u, v) \in E^{2} .
\end{aligned}
$$

Let $m_{0}(t)=\left(K b_{1}\right)(t), n_{0}(t)=\left(K b_{2}\right)(t)$. Then condition $\left(C_{4}\right)$ in Lemma 2.8 is satisfied. 
According to Lemma 2.8, we derive that there exists a sufficiently large number $R>0$ such that

$$
\operatorname{deg}\left(I-A, B_{R}, 0\right)=0 .
$$

From $\left(\mathrm{A}_{4}\right)$, it follows that there exist $0<\varepsilon_{2}<1$ and $0<r<R$ such that

$$
\begin{gathered}
|f(t, x)| \leq\left(1-\varepsilon_{2}\right) \lambda_{1}|x|, \quad|g(t, y)| \leq\left(1-\varepsilon_{2}\right) \lambda_{1}|y|, \\
\forall t \in[0,1], x, y \in \mathbb{R} \text { with }|x| \leq r,|y| \leq r .
\end{gathered}
$$

Thus

$$
\begin{aligned}
& \left|\left(A_{1} u\right)(t)\right| \leq\left(1-\varepsilon_{2}\right) \lambda_{1}(K|v|)(t), \quad\left(A_{2} v\right)(t) \mid \leq\left(1-\varepsilon_{2}\right) \lambda_{1}(K|u|)(t), \\
& \forall t \in[0,1], u, v \in E \text { with }\|u\| \leq r,\|v\| \leq r .
\end{aligned}
$$

Next we will prove that

$$
(u, v) \neq \mu A(u, v) \quad \text { for all }(u, v) \in \partial B_{r} \text { and } \mu \in[0,1] .
$$

If there exist $\left(u_{1}, v_{1}\right) \in \partial B_{r}$ and $\mu_{1} \in[0,1]$ such that $\left(u_{1}, v_{1}\right) \neq \mu A\left(u_{1}, v_{1}\right)$, that is,

$$
\begin{aligned}
& u_{1}(t)=\left(A_{1} v_{1}\right)(t)=\mu_{1} \int_{0}^{1} G(t, s) h(s) f\left(s, v_{1}(s)\right) d s, \\
& v_{1}(t)=\left(A_{2} u_{1}\right)(t)=\mu_{1} \int_{0}^{1} G(t, s) h(s) g\left(s, u_{1}(s)\right) d s .
\end{aligned}
$$

Let $z(t)=\left|u_{1}(t)\right|+\left|v_{1}(t)\right|$. Then $z \in P$ and by (3.4),

$$
\begin{aligned}
z(t) & \leq\left(1-\varepsilon_{2}\right) \lambda_{1}\left[\left(K\left|u_{1}\right|\right)(t)+\left(K\left|v_{1}\right|\right)(t)\right] \\
& =\left(1-\varepsilon_{2}\right) \lambda_{1}\left(K\left(\left|u_{1}\right|+\left|v_{1}\right|\right)\right)(t)=\left(1-\varepsilon_{2}\right) \lambda_{1}(K z)(t) .
\end{aligned}
$$

The $n$th iteration of this inequality shows that $z(t) \leq\left(1-\varepsilon_{2}\right)^{n} \lambda_{1}^{n}\left(K^{n} z\right)(t)(n=1,2, \ldots)$, so $\|z\| \leq\left(1-\varepsilon_{2}\right)^{n} \lambda_{1}^{n}\left\|K^{n}\right\| \cdot\|z\|$, that is, $1 \leq\left(1-\varepsilon_{2}\right)^{n} \lambda_{1}^{n}\left\|K^{n}\right\|$. This yields $1-\varepsilon_{2}=\left(1-\varepsilon_{2}\right) \lambda_{1} r(K)=$ $\left(1-\varepsilon_{2}\right) \lambda_{1} \lim _{n \rightarrow \infty} \sqrt[n]{\left\|K^{n}\right\|} \geq 1$, which is a contradictory inequality. Hence, (3.5) holds.

It follows from (3.5) and Lemma 2.7 that

$$
\operatorname{deg}\left(I-A, B_{r}, 0\right)=1 .
$$

By (3.3), (3.6), and the additivity of Leray-Schauder degree, we obtain

$$
\operatorname{deg}\left(I-A, B_{R} \backslash \bar{B}_{r}, 0\right)=\operatorname{deg}\left(I-A, B_{R}, 0\right)-\operatorname{deg}\left(I-A, B_{r}, 0\right)=-1 .
$$

So $A$ has at least one fixed point on $B_{R} \backslash \bar{B}_{r}$, namely FBVP (1.1) has at least one nontrivial solution. 


\section{Uniqueness}

Theorem 4.1 Assume that $\left(\mathrm{A}_{1}\right)-\left(\mathrm{A}_{4}\right)$ are satisfied. Moreover, the following conditions are satisfied:

$\left(\mathrm{A}_{5}\right) 0<\int_{0}^{1} g_{\beta}(s) h(s) d s<+\infty$ and there exists a constant $k<\left[\int_{0}^{1}\left(\frac{s(1-s)^{\alpha-1}}{\Gamma(\alpha-1)}+\frac{g_{\beta}(s)}{1-\Gamma}\right) h(s) d s\right]^{-1}$ such that

$$
|f(t, x)-f(t, y)| \leq k|x-y|, \quad|g(t, x)-g(t, y)| \leq k|x-y| \quad \text { for any } x, y \in \mathbb{R} .
$$

\section{Then FBVP (1.1) has a unique solution.}

Proof It follows from $|f(t, x)-f(t, y)| \leq k|x-y|,|g(t, x)-g(t, y)| \leq k|x-y|$ for any $x, y \in \mathbb{R}$ that $\left(\mathrm{A}_{1}\right)$ holds. Then by Theorem 3.1, FBVP (1.1) has at least one nontrivial solution. Suppose that FBVP (1.1) has two different solutions $\left(u_{1}(t), v_{1}(t)\right)$ and $\left(u_{2}(t), v_{2}(t)\right)$. By Lemma 2.6, $G(t, s) \leq \frac{s(1-s)^{\alpha-1}}{\Gamma(\alpha-1)}+\frac{g_{\beta}(s)}{1-\Gamma}$. Then from $\left(\mathrm{A}_{5}\right)$ it follows that

$$
\begin{aligned}
\left\|u_{1}-u_{2}\right\| & =\max _{t \in[0,1]}\left|\left(A_{1} v_{1}\right)(t)-\left(A_{1} v_{2}\right)(t)\right| \\
& \leq \max _{t \in[0,1]} \int_{0}^{1} G(t, s) h(s)\left|f\left(s, v_{1}(s)\right)-f\left(s, v_{2}(s)\right)\right| d s \\
& \leq k\left\|v_{1}-v_{2}\right\| \max _{t \in[0,1]} \int_{0}^{1} G(t, s) h(s) d s \\
& \leq k\left\|v_{1}-v_{2}\right\| \int_{0}^{1}\left(\frac{s(1-s)^{\alpha-1}}{\Gamma(\alpha-1)}+\frac{g_{\beta}(s)}{1-\Gamma}\right) h(s) d s \\
& <\left\|v_{1}-v_{2}\right\|, \\
\left\|v_{1}-v_{2}\right\| & =\max _{t \in[0,1]}\left|\left(A_{2} u_{1}\right)(t)-\left(A_{2} u_{2}\right)(t)\right| \\
& \leq \max _{t \in[0,1]} \int_{0}^{1} G(t, s) h(s)\left|f\left(s, u_{1}(s)\right)-f\left(s, u_{2}(s)\right)\right| d s \\
& \leq k\left\|u_{1}-u_{2}\right\| \max _{t \in[0,1]}^{1} G(t, s) h(s) d s \\
& \leq k\left\|u_{1}-u_{2}\right\| \int_{0}^{1}\left(\frac{s(1-s)^{\alpha-1}}{\Gamma(\alpha-1)}+\frac{g_{\beta}(s)}{1-\Gamma}\right) h(s) d s \\
& <\left\|u_{1}-u_{2}\right\| .
\end{aligned}
$$

By adding the above two inequalities, we obtain $\left\|v_{1}-v_{2}\right\|+\left\|u_{1}-u_{2}\right\|<\left\|v_{1}-v_{2}\right\|+\left\|u_{1}-u_{2}\right\|$, which is a contradictory inequality. Therefore $\left(u_{1}(t), v_{1}(t)\right)=\left(u_{2}(t), v_{2}(t)\right)$ and FBVP $(1.1)$ has a unique solution.

\section{Examples}

Example 5.1 Consider FBVP (1.1) with

$$
\beta(t)= \begin{cases}0, & {\left[0, \frac{1}{3}\right),} \\ \frac{1}{8}, & {\left[\frac{1}{3}, \frac{2}{3}\right),} \\ \frac{1}{2}, & {\left[\frac{2}{3}, 1\right],}\end{cases}
$$




$$
\begin{aligned}
h(t)=\frac{1}{\sqrt{t(1-t)}} \text { and } \\
f(t, x)= \begin{cases}\sum_{i=1}^{n}(-1)^{i} a_{i}-\left(1+t^{2}\right)|x|^{\frac{1}{3}} \ln (|x|+1)+\left(1+t^{2}\right) \ln 2, & x \in(-\infty,-1), \\
\sum_{i=1}^{n} a_{i} x^{i}, & x \in[-1,+\infty),\end{cases} \\
g(t, y)= \begin{cases}\sum_{i=1}^{n}(-1)^{i} a_{i}-\left(1+t^{4}\right)|y|^{\frac{1}{3}} \ln \left(|y|^{\frac{1}{3}}+1\right)+\left(1+t^{4}\right) \ln 2, & y \in(-\infty,-1), \\
\sum_{i=1}^{n} a_{i} y^{i}, & y \in[-1,+\infty),\end{cases}
\end{aligned}
$$

where $0<a_{1}<\lambda_{1}, a_{n}>0$. Obviously, $\Gamma=\int_{0}^{1} t^{\alpha-1} d \beta(t)=\frac{1}{8}\left(\frac{1}{3}\right)^{\alpha-1}+\frac{3}{8}\left(\frac{2}{3}\right)^{\alpha-1}<\frac{7}{24}<1$. Then $h$ is singular at $t=0,1$ and $f, g$ are unbounded from below. Take $c_{1}(t)=1+t^{2}, c_{2}(t)=1+t^{4}$, $b_{1}(t)=\sum_{i=1}^{n} a_{i}+\left(1+t^{2}\right) \ln 2, b_{2}(t)=\sum_{i=1}^{n} a_{i}+\left(1+t^{4}\right) \ln 2, B_{1}(x)=|x|^{\frac{1}{3}} \ln (|x|+1), B_{2}(x)=$ $|x|^{\frac{1}{3}} \ln \left(|x|^{\frac{1}{3}}+1\right)$. Then all the conditions in Theorem 3.1 are satisfied. Therefore, FBVP (1.1) with the above $\beta(t), h(t), f(t, x), g(t, y)$ has at least one nontrivial solution.

Example 5.2 Consider FBVP (1.1) with

$$
\begin{aligned}
& \beta(t)= \begin{cases}0, & {\left[0, \frac{1}{3}\right),} \\
\frac{1}{3}, & {\left[\frac{1}{3}, 1\right],}\end{cases} \\
& f(t, x)=g(t, x)= \begin{cases}-a_{1}-\left(1+t^{2}\right) \ln (|x|+1)+\left(1+t^{2}\right) \ln 2, & x \in(-\infty,-1), \\
a_{1} x, & x \in[-1,1), \\
a_{2}+a_{2} \ln (x+1)+a_{1}-a_{2}-a_{2} \ln 2, & x \in[1,+\infty) .\end{cases}
\end{aligned}
$$

$h(t)=\frac{\Gamma(\alpha-1)}{2\left(5 a_{2}+2\right) \sqrt{t}(1-t)^{\alpha-1}}$, where $0<a_{1}<\lambda_{1}<a_{2}$.

Take $c_{i}(t)=1+t^{2}, b_{i}(t)=a_{1}+\left(1+t^{2}\right) \ln 2, B_{i}(x)=\ln (|x|+1), i=1,2$. Then $\left(\mathrm{A}_{2}\right)$ is satisfied. The choice of $a_{1}, a_{2}$ guarantees that $\left(\mathrm{A}_{3}\right)$ and $\left(\mathrm{A}_{4}\right)$ are satisfied. By some simple computation, we obtain that $\Gamma=\int_{0}^{1} t^{\alpha-1} d \beta(t)=\frac{1}{3^{\alpha}}<1,|f(t, x)-f(t, y)| \leq\left(a_{1}+\frac{3}{2} a_{2}+1\right)|x-y|$, $|g(t, x)-g(t, y)| \leq\left(a_{1}+\frac{3}{2} a_{2}+1\right)|x-y|$ for any $x, y \in \mathbb{R}$ and $\int_{0}^{1}\left(\frac{s(1-s)^{\alpha-1}}{\Gamma(\alpha-1)}+\frac{g_{\beta}(s)}{1-\Gamma}\right) h(s) d s<\frac{4}{3\left(5 a_{2}+2\right)}$. Hence $\left(\mathrm{A}_{5}\right)$ holds. So FBVP $(1.1)$ with the above $\beta(t), h(t), f(t, x), g(t, y)$ has a unique solution.

\section{Acknowledgements}

The authors are grateful to editors and reviewers for their valuable suggestions.

\section{Funding}

This paper is supported by the National Natural Science Foundation of China (11701252) and the Natural Science Foundation of Shangdong Province of China (ZR2017MA036).

\section{Competing interests}

The authors declare that they have no competing interests.

\section{Authors' contributions}

All authors contributed equally to the writing of this paper. All authors read and approved the final manuscript.

\section{Author details}

'School of Mathematics, China University of Mining and Technology, Xuzhou, China. ${ }^{2}$ School of Mathematical Sciences, Qufu Normal University, Qufu, China.

\section{Publisher's Note}

Springer Nature remains neutral with regard to jurisdictional claims in published maps and institutional affiliations. 


\section{References}

1. Podlubny, l.: Fractional Differential Equations. Mathematics in Science and Engineering, vol. 198. Academic Press, New York (1999)

2. Kilbas, A.A., Srivastava, H.M., Trujillo, J.J.: Theory and Applications of Fractional Differential Equations. Elsevier, Amsterdam (2006)

3. Diethelm, K.: The Analysis of Fractional Differential Equation. Springer, Heidelberg (2010)

4. Bai, C., Fang, J.: The existence of a positive solution for a singular coupled system of nonlinear fractional differential equations. Appl. Math. Comput. 150, 611-621 (2004)

5. Su, X.: Boundary value problem for a coupled system of nonlinear fractional differential equations. Appl. Math. Lett. 22, 64-69 (2009)

6. Ahmad, B., Nieto, J.: Existence results for a coupled system of nonlinear fractional differential equations with three-point boundary conditions. Comput. Math. Appl. 58, 1838-1843 (2009)

7. Wang, W.: Positive solutions for a coupled system of nonlinear fractional differential equations with integral boundary conditions. Comput. Math. Appl. 63, 288-297 (2012)

8. Li, Y., Li, F.: Sign-changing solutions to second-order integral boundary value problems. Nonlinear Anal. 69 , 1179-1187 (2008)

9. Yang, Z.: Existence of nontrivial solutions for a nonlinear Sturm-Liouville problem with integral boundary value conditions. Nonlinear Anal. 68, 216-225 (2008)

10. Boucherif, A.: Second-order boundary value problems with integral boundary conditions. Nonlinear Anal. 70, 364-371 (2009)

11. Liu, L., Liu, B., Wu, H.: Nontrivial solutions of $m$-point boundary value problems for singular second-order differential equations with a sign-changing nonlinear term. J. Comput. Appl. Math. 224, 373-382 (2009)

12. Jiang, J., Liu, L., Wu, Y.: Second-order nonlinear singular Sturm-Liouville problems with integral boundary conditions. Appl. Math. Comput. 215, 1573-1582 (2009)

13. Kong, L.: Second order singular boundary value problems with integral boundary conditions. Nonlinear Anal. 72, 2628-2638 (2010)

14. Graef, J.R., Kong, L.: Periodic solutions for functional differential equations with sign-changing nonlinearities. Proc. R. Soc. Edinb. 140A, 597-616 (2010)

15. Chamberlain, J., Kong, L., Kong, Q.: Nodal solutions of boundary value problems with boundary conditions involving Riemann-Stieltjes integrals. Nonlinear Anal. 74, 2380-2387 (2011)

16. Li, H., Liu, Y.: On sign-changing solutions for a second-order integral boundary value problem. Comput. Math. Appl. 62, 651-656 (2011)

17. Feng, M.: Existence of symmetric positive solutions for a boundary value problem with integral boundary conditions Appl. Math. Lett. 24, 1419-1427 (2011)

18. Wang, Y., Liu, L., Wu, Y.: Positive solutions for a class of fractional boundary value problem with changing sign nonlinearity. Nonlinear Anal. 74, 6434-6441 (2011)

19. Wang, Y., Liu, L., Wu, Y.: Positive solutions for a nonlocal fractional differential equation. Nonlinear Anal. 74, 3599-3605 (2011)

20. Chen, T., Liu, W., Hu, Z.: A boundary value problem for fractional differential equation with $p$-Laplacian operator at resonance. Nonlinear Anal. 75, 3210-3217 (2012)

21. Liu, B., Li, J., Liu, L.: Nontrivial solutions for a boundary value problem with integral boundary conditions. Bound. Value Probl. 2014, 15 (2014)

22. Li, H., Liu, L., Wu, Y.: Positive solutions for singular nonlinear fractional differential equation with integral boundary conditions. Bound. Value Probl. 2015, 232 (2015)

23. Wang, Y., Liu, L.: Necessary and sufficient condition for the existence of positive solution to singular fractional differential equations. Adv. Differ. Equ. 2015, 207 (2015)

24. Jiang, J., Liu, L.: Existence of solutions for a sequential fractional differential system with coupled boundary conditions. Bound. Value Probl. 2016, 159 (2016)

25. Hao, X., Wang, H., Liu, L., Cu, Y.: Positive solutions for a system of nonlinear fractional nonlocal boundary value problems with parameters and p-Laplacian operator. Bound. Value Probl. 2017, 18 (2017)

26. Bai, Z., Lü, H.: Positive solutions for boundary value problem of nonlinear fractional differential equation. J. Math. Anal. Appl. 311, 495-505 (2005)

27. Bai, Z., Chen, Y., Lian, H., Sun, S.: On the existence of blow up solutions for a class of fractional differential equations. Fract. Calc. Appl. Anal. 17, 1175-1187 (2014)

28. Bai, Z:: On positive solutions of a nonlocal fractional boundary value problem. Nonlinear Anal. 72, 916-924 (2010)

29. Bai, Z., Zhang, S., Sun, S., Yin, C.: Monotone iterative method for fractional differential equations. Electron. J. Differ. Equ. 2016, 6 (2016)

30. Song, Q., Dong, X., Bai, Z., Chen, B.: Existence for fractional Dirichlet boundary value problem under barrier strip conditions. J. Nonlinear Sci. Appl. 10, 3592-3598 (2017)

31. Yuan, C.: Multiple positive solutions for $(n-1,1)$-type semipositone conjugate boundary value problems of nonlinear fractional differential equations. Electron. J. Qual. Theory Differ. Equ. 2010, 36 (2010)

32. Webb, J.R.L., Lan, K.: Eigenvalue criteria for existence of multiple positive solutions of nonlinear boundary value problems of local and nonlocal type. Topol. Methods Nonlinear Anal. 27, 91-115 (2006)

33. Krein, M.G., Rutman, M.A.: Linear operators leaving invariant a cone in a Banach space. Transl. Am. Math. Soc. 26 199-325 (1950)

34. Guo, D., Lakshmikantham, V.: Nonlinear Problems in Abstract Cones. Academic Press, Orlando (1988)

35. Ahmad, B., Luca, R.: Existence of solutions for a system of fractional differential equations with coupled nonlocal boundary conditions. Fract. Calc. Appl. Anal. 21, 423-441 (2018)

36. Agarwal, R.P., Ahmad, B., Garout, D.: Existence results for coupled nonlinear fractional differential equations equipped with nonlocal coupled flux and multi-point boundary conditions. Chaos Solitons Fractals 102, 149-161 (2017)

37. Ahmad, B., Alsaedi, A., Aljoudi, S.: On a coupled system of sequential fractional differential equations with variable coefficients and coupled integral boundary conditions. Bull. Math. Soc. Sci. Math. Roum. 60, 3-18 (2017) 